\title{
CICLOS DE AQUECIMENTO-PULSO DE VÁCUO PARA MODIFICAÇÃO DA TEXTURA DE BANANAS DESIDRATADAS EM CAMPO DE MICRO-ONDAS
}

\author{
R. L. MONTEIRO ${ }^{1}$, B. A. M. CARCIOFI ${ }^{1}$ e J. B. LAURINDO ${ }^{1}$ \\ ${ }^{1}$ Universidade Federal de Santa Catarina, Departamento de Engenharia Química e Engenharia de \\ Alimentos \\ E-mail para contato: joao@enq.ufsc.br
}

\begin{abstract}
RESUMO - Este trabalho avaliou a influência da aplicação de múltiplos ciclos de aquecimento-pulso de vácuo (MFD) nas propriedades físicas que controlam a crocância de frutas desidratadas em campo de micro-ondas. Fatias de bananas (var. Prata) de $5 \mathrm{~mm}$ de espessura foram desidratadas pela secagem com: I) micro-ondas a vácuo MV, e II) MFD, associados a um período complementar de secagem por micro-ondas sob vácuo (MVMFD). As amostras secas pelo MV apresentaram umidade em base seca e atividade de água de $0,0341 \mathrm{~g} \mathrm{~g}^{-1}$ e 0,259 , respectivamente, enquanto que para o MVMFD, esses valores foram $0,0518 \mathrm{~g} \mathrm{~g}^{-1}$ e 0,393. As micrografias mostraram a formação de poros maiores nas amostras MVMFD, além de maior porosidade (55\% contra $43 \%$ ). Isso implicou em maior irregularidade na curva de força x penetração dessas amostras, indicando maior crocância do produto final. Os resultados obtidos mostram que é possível controlar a textura das frutas desidratadas através da aplicação MFD, em campo de micro-ondas.
\end{abstract}

\section{INTRODUÇÃO}

A banana é um fruto climatérico, altamente perecível, apresentando elevada taxa respiratória e de produção de etileno, conduzindo rapidamente ao amadurecimento (Pinheiro et $a l .$, 2007). Estima-se que as perdas na cadeia produtiva da banana brasileira são de até $40 \%$. As principais causas são as técnicas inadequadas de colheita e pós-colheita, as quais comprometem a qualidade do produto. Falhas na distribuição e a dificuldade na colocação do produto no mercado também podem ser apontadas como causas de perdas (Chitarra e Chitarra, 2005).

Uma alternativa para diminuir os desperdícios de banana é a desidratação, como forma de reduzir a atividade de água (aw) das frutas para inibir o crescimento microbiano, retardando a deterioração das mesmas. Desta forma, é possível o aumento da vida de prateleira dos alimentos, a redução do peso e do volume a serem transportados, facilitando e reduzindo os custos, além de facilitar o consumo e diversificar a oferta de produtos.

Os processos CMFD (convective multi-flash drying) e KMFD (condutive multi-flash drying) desidratam e ao mesmo tempo proporcionam uma textura crocante as frutas. O processo 


\section{9 a 22 de outubro de 2014 \\ Florianópolis/SC}

baseia-se na aplicação de sucessivos ciclos de aquecimento e de pulsos de vácuo. O produto é aquecido à pressão atmosférica usando ar quente (aquecimento convectivo) ou uma superfície aquecida (aquecimento condutivo). Quando o produto atinge a temperatura desejada, uma redução súbita de pressão (pulso de vácuo) é aplicada, o que leva a evaporação de água (flash) e consequente resfriamento do produto (Laurindo et al., 2011).

A secagem por micro-ondas apresenta como principal vantagem à rápida taxa de remoção de água, podendo resultar em produtos secos de qualidades nutricionais e sensoriais elevadas. As ondas eletromagnéticas promovem o aquecimento uniforme, dessa forma, vapores são formados no interior do produto, gerando um gradiente interno de pressão, que irá forçar a saída da água. Assim, o encolhimento dos materiais alimentares é diminuído. $\mathrm{O}$ aquecimento pelas micro-ondas combinado à aplicação de vácuo pode melhorar a eficiência da secagem, bem como a qualidade dos produtos alimentares. Ainda como consequência, a secagem a vácuo impede a oxidação devido à ausência de ar, e, consequentemente, mantém a cor, textura e sabor dos produtos secos (Zhang et al., 2006).

O objetivo do presente trabalho foi produzir fatias de banana crocantes obtidas pela aplicação de múltiplos pulsos sequenciais de vácuo durante o processo de secagem por microondas e relacionar as condições de processo com parâmetros analíticos de textura.

\section{MATERIAL E MÉTODOS}

\subsection{Seleção e preparação da matéria-prima}

As bananas da variedade Prata (Musa Sapientum L.) foram adquiridas no comércio local da cidade de Florianópolis - SC e mantidas a temperatura ambiente até o momento da sua utilização. A seleção das frutas foi realizada pelo grau de maturação, através de análise visual, resistência à penetração e conteúdo de sólidos solúveis (SST). A resistência à penetração foi avaliada com o auxílio de um penetrômetro (Effegi Modelo - FT 327, $\varnothing=8 \mathrm{~mm}$, Itália) e o teor de SST foi medido por um refratômetro óptico com resolução 0,1 ${ }^{\circ}$ Brix (Pocket refractometer Atago, Modelo - PAL-BX/RI, Japão). As bananas foram descascadas manualmente e cortadas em fatias de $5 \mathrm{~mm}$ de espessura em dispositivo específico para este fim.

\subsection{Dispositivo experimental}

As secagens foram realizadas em um forno doméstico adaptado (Electrolux, modelo MEX55, Brasil) com espaço interno de 45 litros, potência máxima de saída do magnetron de $1000 \mathrm{~W}$ e frequência de $2450 \mathrm{MHz}$. No interior do forno foi adaptado um recipiente de polipropileno que serviu como câmara de vácuo. Este recipiente foi conectado a uma bomba de vácuo e a um transdutor de pressão (Warme, Modelo - WTP4010, Brasil) que enviou os dados para registro em um computador. Um sistema consistindo de uma válvula $\mathrm{T}$ conectada a uma junta rotativa formada por um eixo fixo (conectado à bomba de vácuo e ao transdutor de pressão) e por um eixo livre (conectado à câmara de vácuo) permitiu homogeneizar a distribuição das micro-ondas pelo acionamento do motor do prato giratório típico dos fornos de micro-ondas 
domésticos, o dispositivo experimental está representado na Figura 1.

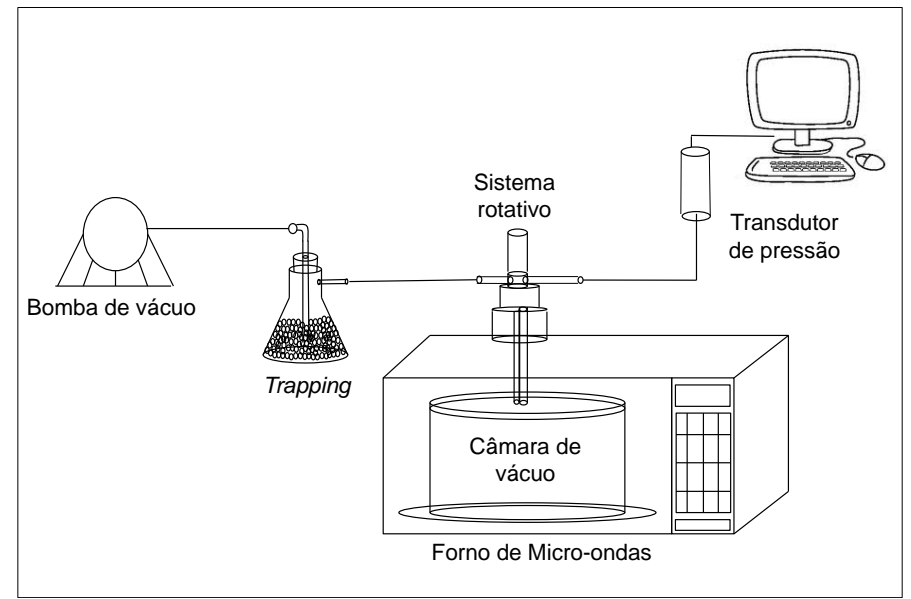

Figura 1 - Dispositivo experimental.

\subsection{Secagem por Micro-ondas}

Para a secagem, as fatias de banana foram dispostas ao redor da extremidade de uma bandeja circular de polipropileno inserida na câmara de vácuo. $\mathrm{O}$ forno micro-ondas, quando ligado, esteve na potência máxima do magnetrom $(1000 \mathrm{~W})$.

Foram adotados dois procedimentos de secagem (Figura 2): I) Micro-ondas a vácuo (MV), inicialmente reduziu-se a pressão em aproximadamente $4,0 \mathrm{kPa}$, neste instante o micro-ondas foi ligado por 12 min e então restabeleceu-se a pressão atmosférica, totalizando 13,67 min de processo e II) Múltiplos ciclos de aquecimento-pulso de vácuo (MFD), associados a um período complementar de secagem por micro-ondas sob vácuo (MVMFD), totalizando 16,52 min. No processo MVMFD aqueceu-se a amostra no micro-ondas por $30 \mathrm{~s}$ (tempo para que a amostra atingisse $60{ }^{\circ} \mathrm{C}$ ), quando foi aplicado o pulso de vácuo até a pressão final de $8,0 \mathrm{kPa}$, esse procedimento foi repetido por mais duas vezes, sendo a duração do aquecimento por micro-ondas de $20 \mathrm{~s}$. 


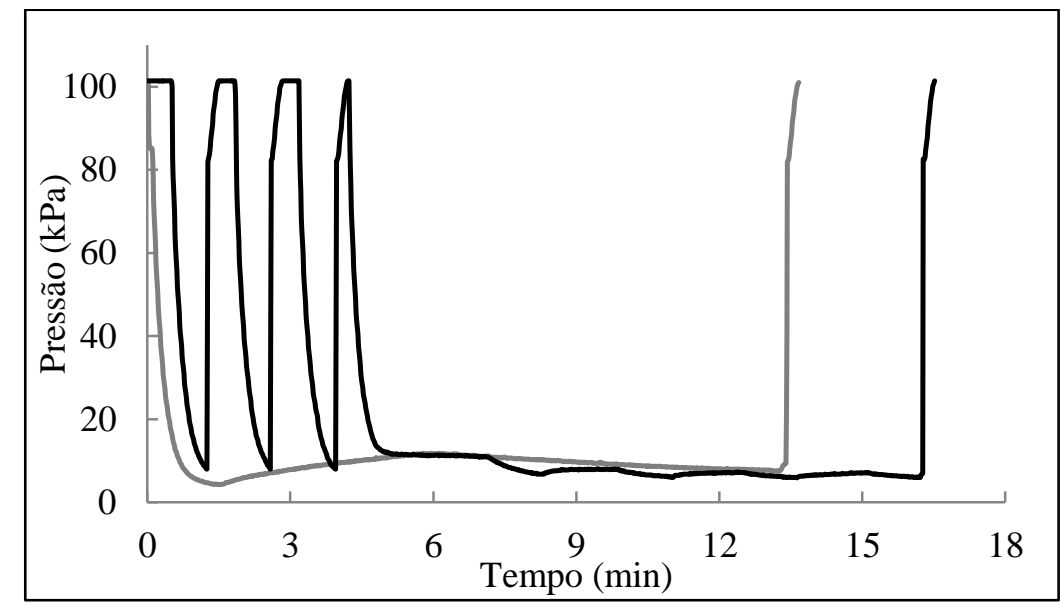

Figura 2 - Evolução temporal da pressão na secagem MV (-) e MVMFD (-) para potência de $1000 \mathrm{~W}$.

\subsection{Caracterização físico-química}

As frutas in natura foram caracterizadas pelo conteúdo de água, de sólidos solúveis totais (SST) e pela atividade de água (aw). No decorrer da secagem, as amostras foram caracterizadas por: umidade, aw, volume aparente, volume real, porosidade, teste de penetração e microscopia eletrônica de varredura (MEV). As metodologias utilizadas são descritas a seguir.

O conteúdo de água foi determinado pelo método gravimétrico (Shimadzu, Modelo AY220, Filipinas com precisão de $0,0001 \mathrm{~g})$ a vácuo $\left(\leq 100 \mathrm{mmHg}, 70{ }^{\circ} \mathrm{C}\right.$ ) conforme metodologia da AOAC (2005). As determinações foram realizadas em quadruplicata.

A atividade de água (aw) foi determinada em higrômetro digital (Aqualab Modelo - Series 3 TE, Decagon Devices, Inc., Pullman, EUA) pela medida do ponto de orvalho a $25{ }^{\circ} \mathrm{C}$. As análises foram realizadas em triplicata.

$\mathrm{O}$ volume aparente $\left(\mathrm{V}_{\mathrm{ap}}\right)$ foi determinado pela força de empuxo da amostra imersa em nheptano. A amostra foi presa em um gancho conectado à parte inferior de uma balança analítica com precisão de 0,0001 g (Marte, Modelo -AY220, Filipinas). O V ap foi calculado pela Equação 1 , sendo a análise realizada em quadruplicata.

$$
V_{a p}=\frac{\left(m_{s+g}-m_{g}\right)-\left(m_{s+g, n-h p}-m_{g \cdot n-h p}\right)}{\rho_{n-h p}}
$$

na qual $m_{\mathrm{g}}$ é massa do gancho, $m_{\mathrm{s}+\mathrm{g}}$ é a massa da amostra e do gancho, $m_{\mathrm{s}+\mathrm{g}, \mathrm{n}-\mathrm{hp}}$ é a massa

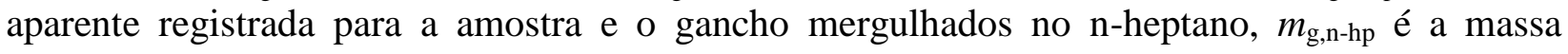
aparente registrada para gancho mergulhado no n-heptano.

$\mathrm{O}$ volume real $\left(\mathrm{V}_{\mathrm{r}}\right)$ da amostra foi determinado utilizando um picnômetro de ar 
comprimido, esquematizado na Figura 3.

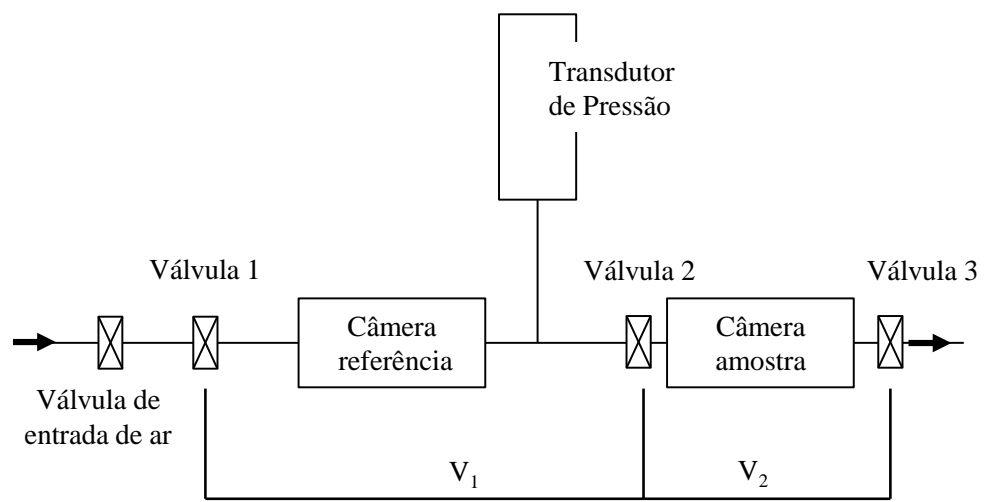

Figura 3 - Diagrama esquemático do picnômetro (Adaptado de Sereno et al., 2007).

$\mathrm{O}$ procedimento realizado foi conforme proposto por Sereno et al. (2007). O $\mathrm{V}_{\mathrm{r}}$ foi calculado utilizando a Equação 2, sendo a análise realizada em quadruplicata.

$$
V_{r}=\frac{V_{1}\left(\frac{P_{1}}{P_{2}} \frac{T_{2}}{T_{1}}-1\right)}{\left(\frac{P_{a t m}}{P_{2}} \frac{T_{2}}{T_{a m b}}-1\right)}+V_{2}
$$

na qual é $P_{a t m}$ e $T_{a m b}$ são a pressão e a temperatura medida com picnómetro vazio e a válvula 1 fechada. $P_{1}$ e $T_{1}$ são a pressão e a temperatura medida após o equilíbrio da compressão de ar na câmera de referência, com as a válvula 1,2 e 3 fechadas. $P_{2}$ e $T_{2}$ são a pressão e a temperatura medida após a abertura da válvula 2, com as a válvula 1 e 3 fechadas.

A porosidade $(\varepsilon)$ das amostras foi calculada a partir dos valores de $V_{r}$ e $V_{a p}$, conforme a Equação 3.

$$
\varepsilon=\left(1-\frac{V_{r}}{V_{a p}}\right) \cdot 100
$$

Os teste de penetração (puncture test) foram realizados com probe cilíndrica de $2 \mathrm{~mm}$ de diâmetro em um analisador de textura (Stable Micro System, Modelo - TA-HD-Plus, Reino Unido). Ao final de cada processo de desidratação foram analisadas 5 amostras, sendo realizadas 3 perfurações em diferentes regiões das mesmas.

Para as imagens de MEV (Modelo - JEOL JSM 6390LV, Japão) as amostras desidratadas foram liofilizadas (Liobras, Modelo - Liotop L101, Brasil) durante 24 horas, para a retirada da umidade residual, e recobertas com ouro utilizando um metalizador (LEICA, Modelo - EM SCD500). As imagens capturadas foram da parte interna das amostras fraturadas. 


\section{RESULTADOS E DISCUSSÃO}

As amostras de banana in natura utilizadas nos processos de secagem apresentaram a concentração de SST de 23,1 $\pm 1,2{ }^{\circ}$ Brix (média \pm desvio padrão), resistência à penetração de $1,2 \pm 0,1 \mathrm{kgf} \mathrm{cm}^{-2}$ e a massa de banana utilizada na secagem foi de $85,80 \pm 0,82 \mathrm{~g}$.

As Figuras 4 e 5 apresentam os dados em triplicata da evolução temporal da umidade em base seca (Xbs) e da atividade de água $\left(\mathrm{a}_{\mathrm{w}}\right)$ das amostras durante as secagens por MV e MVMFD e apresentam uma boa reprodutibilidade da evolução temporal da Xbs e $\mathrm{a}_{\mathrm{w}}$ das amostras durante as secagens, mesmo com a amostragem realizada de forma destrutiva. As bananas in natura apresentaram umidade em base seca média ( \pm desvio padrão) de $2,4365( \pm 0,1195) \mathrm{g} \mathrm{g}^{-1}$ e ao final dos processos MV e MVMFD estes valores foram reduzidos para 0,0341 $( \pm 0,0025)$ e $0,0518( \pm 0,0128) \mathrm{g} \mathrm{g}^{-1}$, respectivamente. A atividade de água inicial média ( \pm desvio padrão) foi de $0,981( \pm 0,005)$ e ao final dos processos MV e MVMFD atingiram 0,259 $( \pm 0,006)$ e $0,393( \pm 0,012)$, respectivamente.

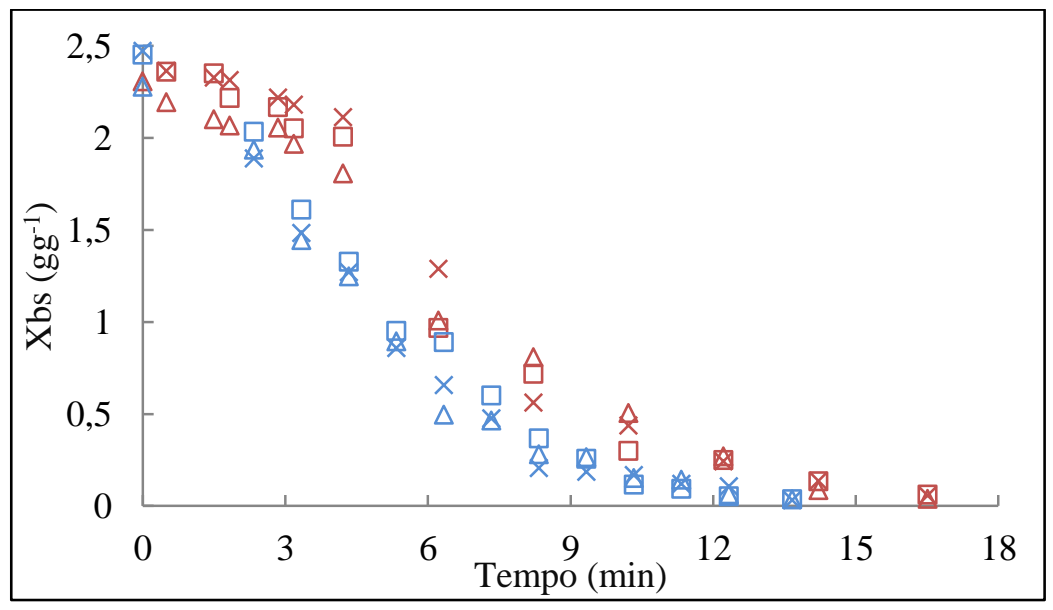

Figura 4 - Evolução temporal da umidade em base seca das amostras de banana durante os processos de secagem MV (-) e MVMFD (-), realizada em triplicata.

A secagem MVMFD resultou na formação de microestrutura diferenciada, indicando uma textura do alimento de característica crocante. Esta textura é verificada pela relação força $\mathrm{x}$ penetração relativa (Figura 6). Resultados semelhantes foram obtidos por Zotarelli et al. (2012), que compararam bananas secas pelo processo CMFD e por liofilização. As frutas CMFD e frutas liofilizadas apresentaram comportamento irregular para os dados obtidos na análise de força $\mathrm{x}$ penetração relativa, semelhantes ao observado no processo MVMFD. 


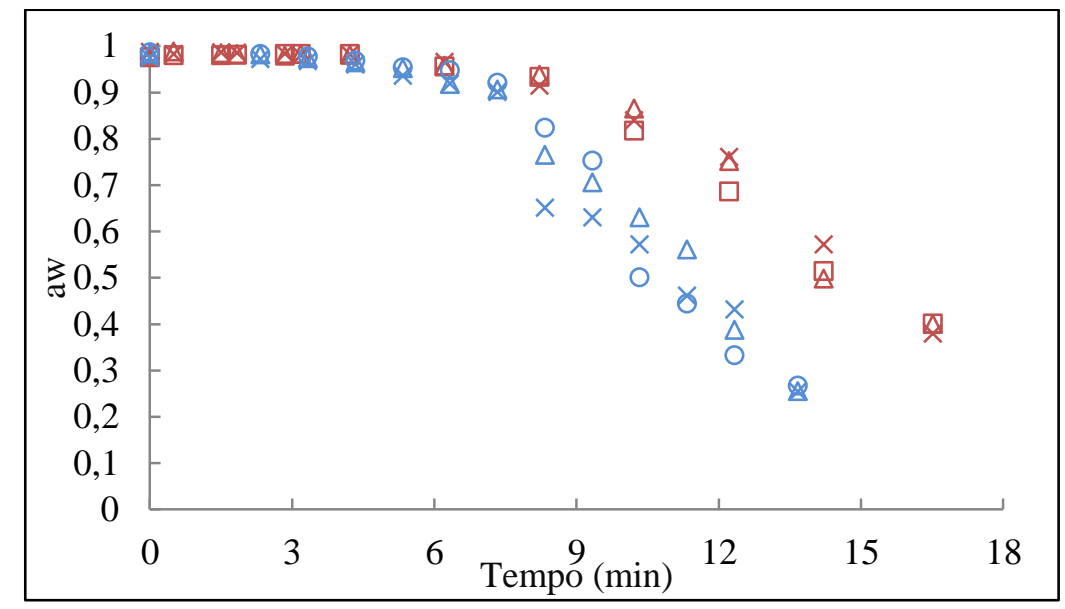

Figura 5 - Evolução temporal da atividade de água das amostras de banana durante os processos de MV (-) e MVMFD (-), realizada em triplicata.

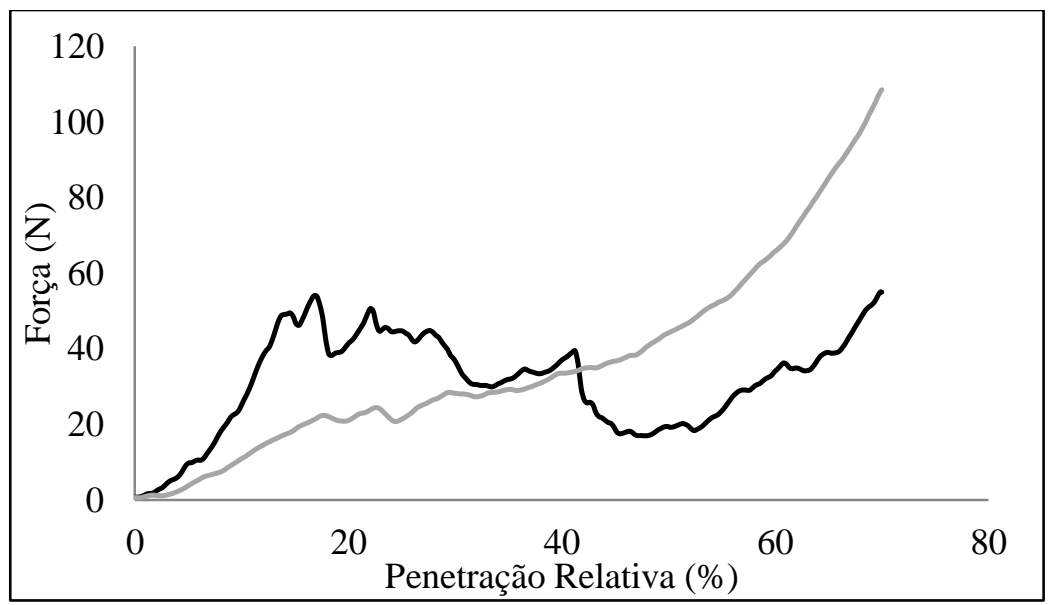

Figura 6 - Relação força-penetração para as fatias de banana secas por MV (-) e MVMFD (一).

As imagens de MEV (Figura 7) permitem observar que o processamento com MVMFD causou uma abertura (expansão) na estrutura interna do produto, formando espaços maiores. Consequentemente, a porosidade da amostra MVMFD $(\varepsilon=55,3 \pm 4,5)$ foi $28 \%$ maior que a porosidade da amostra seca apenas pelo processo MV $(\varepsilon=43,4 \pm 5,4)$.
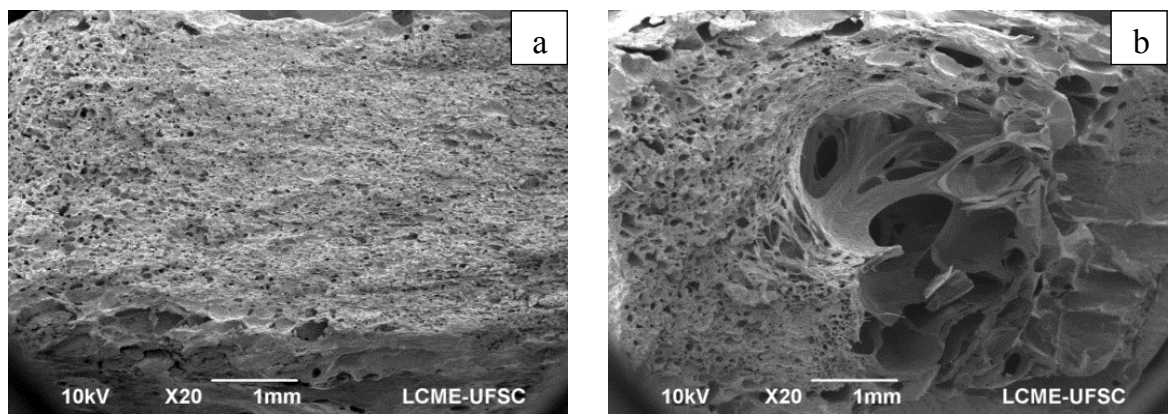

Figura 7 - Imagens de MEV pelo processo (a) MV (b) MVMFD. 


\section{CONCLUSÃO}

É possível alterar a textura das frutas desidratadas através da aplicação de múltiplos ciclos de aquecimento-pulso de vácuo em campo de micro-ondas. O processo de secagem combinando o aquecimento por micro-ondas e sucessivos pulsos de vácuo pode ser competitivo com o processo de liofilização para a produção de frutas desidratadas crocantes, pois pode apresentar frutas com microestrutura semelhante e um menor tempo de processamento. No entanto, dependendo das características da fruta, esta pode passar por deformações irreversíveis, pois o aquecimento das micro-ondas ocorre em todo o material, o que gera um gradiente de pressão interna, resultando em uma força para a saída da água. Deste modo, são necessários estudos detalhados das condições de processo e das características da matéria prima para definir a aplicabilidade e as limitações do processo.

\section{REFERÊNCIAS BIBLIOGRÁFICAS}

AOAC, Official Methods of Analysis, Association of Official Analytical Chemists, Washington, 2005.

CHITARRA, M.I.F.; CHITARRA, A.B. Pós-colheita de frutas e hortaliças: fisiologia $e$ manuseio. 2 ed., Lavras: Editora UFLA, p.785, 2005.

LAURINDO, J.B.; PORCIUNCULA, B.D.A.; ZOTARELLI, M.F. Processo de secagem por sucessivos ciclos de aquecimento-pulso de vácuo (CAPV) para obtenção de alimentos desidratados crocantes. Brazilian Patent PI 017110000045 filed January 24, 2011.

PINHEIRO, A.C.M.; VILAS BOAS, E.V.B.; ALVES, A.P.; SELVA, M.L. Amadurecimento de bananas 'Maçã' submetidas ao 1-metilciclopropeno. Ver. Bras. Frutic., v.29, p.1-4, 2007.

SERENO A. M.; SILVA M.A.; MAYOR, L. Determination of particle density and porosity in foods and porous materials with high moisture content. Int. J. Food Prop., v.10, p. 455-469, 2007.

ZHANG, M.; TANG, J.; MUJUMDAR, A. S.; WANG, S. Trends in microwave-related drying of fruits and vegetables. Trends. Food Sci. Ttech., v.17, p. 524-534, 2006.

ZOTARELLI, M. F.; PORCIUNCULA, B. D. A.; LAURINDO, J. B. A convective multi-flash drying process for producing dehydrated crispy fruits. J. Food Eng., v. 08, p. 523- 531, 2012. 